\title{
Limits on the Magnetic Flux of a Pre-Main Sequence Star
}

\author{
G. Basri ${ }^{1}$ and G.W. Marcy ${ }^{2}$ \\ ${ }^{1}$ Astronomy Department, University of California, Berkeley \\ ${ }^{2}$ Dept. of Physics and Astronomy, San Francisco State University
}

\begin{abstract}
We attempt to detect a magnetic field on the weak T Tauri star, TAP35, via the enhanced equivalent widths of Zeeman-broadened absorption lines. We synthesize $25 \mathrm{Fe} I$ lines, having a range of Zeeman sensitivities, using an LTE Stokes line-transfer calculation. The oscillator strengths of all lines are empirically determined a priori using the same line-transfer code applied to the spectrum of the magnetically quiet star, $\tau$ Ceti. The Fe abundance of TAP35 was established by synthesizing lines that are insensitive to Zeeman splitting. We find that the equivalent widths, $W_{\text {eq }}$, of Zeeman-sensitive lines in TAP35 are systematically enhanced relative to the Zeeman-insensitive lines, consistent with the presence of widespread, kilogauss fields. The excess $W_{\text {eq }}$ can be explained by a product of field strength and surface filling factor $(B f)$ of 1 kiloGauss. A strong upper limit can be placed on the product of those two quantities, $B f<2 \mathrm{kG}$. This measurement bears on the physics of $\mathrm{T}$ Tauri coronae, chromospheres, dynamos, and accretion-disk boundary layers.
\end{abstract}

\section{Introduction}

Pre-main sequence, solar-mass stars exhibit many characteristics suggestive of surface magnetic fields. Spectroscopically, T Tauri stars (TTS) exhibit a wide variety of emission lines such as $\mathrm{H} \alpha, \mathrm{Ca}$ II $\mathrm{H}$ and $\mathrm{K}$ and Fe II at optical wavelengths, as well as lines of $\mathrm{Mg}$ II, Si I, C IV, Si IV, and He II in the UV, all reminiscent of the spectrum of active magnetic regions on the sun (cf. Herbig, 1970; Imhoff and Giampapa, 1982). These lines form above the photosphere in gas at $10^{4}$ to $10^{5} \mathrm{~K}$ that is heated by some non-radiative process, perhaps related to magnetic fields.

Several efforts failed to reproduce theoretically the width and strength of these lines, notably broad $\mathrm{H} \alpha$, Ca II and Mg II, in the context of a "deep chromosphere" (Cram, 1979; Calvet et al., 1984), thus casting doubt on magnetic fields as the energy source for the heating. Doubt also arises owing to the lack of correlation between $\mathrm{H} \alpha$ emission and rotation in TTS as would be expected if a dynamo generates the magnetic fields (Hartmann et al., 1986). Clearly, lines of $\mathrm{H} \alpha$, Ca II, and $\mathrm{Mg}$ II, when broad $\left(\sim 500 \mathrm{~km} \mathrm{~s}^{-1}\right)$, originate in a more extended circumstellar region.

However, in the weak-emission T Tauri Stars (WTTS) the emission lines are narrow $\left(<50 \mathrm{~km} \mathrm{~s}^{-1}\right)$ and deep chromosphere models are successful in reproducing the observed line fluxes and continua. The WTTS UV line fluxes are typically 
10-50 times solar levels, suggesting a corresponding enhancement in the magnetic fields on WTTS relative to the Sun, on which only about $2 \%$ of the surface is covered by kiloGauss fields. Thus one might expect TTS to contain kiloGauss magnetic fields covering a large fraction of their surfaces. Other indicators of surface magnetic fields on TTS include their X-ray properties (Walter, 1987), optical photometric variations, suggestive of starspots (e.g. Vrba et al., 1989), and "flares" (Cohen and Beiging, 1986). Magnetic fields on TTS have been invoked to explain local phenomena such as Alfvén waves (Hartmann et al., 1982), angular momentum loss (Frank Shu, private comm.) and boundary-layer turbulence (Basri, 1990).

Despite the many signatures of widespread surface magnetic fields on TTS, fields have not been detected. Spectropolarimetric efforts have been unsuccessful, perhaps because the fields have opposite polarities locally on the surface, thereby suppressing the net longitudinal field (Brown and Landstreet, 1981; Johnstone and Penston, 1986, 1987). One cannot detect Zeeman broadening of photospheric lines because of the dominance of rotational broadening $\left(v \sin i \sim 20 \mathrm{~km} \mathrm{~s}^{-1}\right)$, though this approach may work if a bright, pole-on TTS could be found.

Here we implement a Zeeman technique that makes use of the enhancement of the equivalent widths of absorption lines owing to the splitting of the lineabsorption coefficient. The technique involves observing absorption lines of different Zeeman sensitivities in the WTTS, TAP35, and in the comparison star, $\tau$ Ceti, in an effort to detect systematic enhancement of the Zeeman-sensitive lines.

\section{Observations}

Spectra were obtained with the Lick Observatory coude echelle spectrometer ("Hamilton") and CCD which has a resolution of 40,000 and has a wavelength coverage from $5000 \AA$ to $8600 \AA$. This setting includes some lines used in past Zeeman work, notably, $\lambda 8468$ and $\lambda 6173$, as well as several lines having low Landé $g$ values. The target TTS, TAP35 (=NTTS $042417+1744, \mathrm{~V}=11$, Walter et al., 1988) was chosen because it has both weak $\mathrm{H} \alpha$ and no IR excess. Thus its absorption lines are not contaminated by emission from a stellar wind or circumstellar disk. In choosing TAP35, we have avoided selection criteria that would bias against the presence of magnetic fields. Its rotation velocity $\left(v \sin i=17 \mathrm{~km} \mathrm{~s}^{-1}\right)$ falls near the average for TTS (Hartmann et al., 1986) as does its X-ray luminosity (Walter et al., 1988).

Four exposures, totaling 3.6 hours, were obtained on the $3-\mathrm{m}$ telescope for TAP35, yielding a final $\mathrm{S} / \mathrm{N}$ ratio in the summed spectrum of $\sim 100: 1$, slightly higher in the near IR than in the yellow region. An exposure of the chromospherically quiet star, $\tau$ Ceti (G8V) was obtained with the same setting. The data were reduced in a standard way: the raw CCD images were divided by normalized flat-field exposures of an incandescent lamp, corrected for scattered light by subtracting the signal between orders, mashed into 54 individual spectral orders, and wavelength-calibrated using exposures of a Th-Ar lamp. 
We searched the entire echelle format for Fe I absorption lines amenable to precise measurement of equivalent width, rejecting lines that were either too weak or blended. This task was complicated by the rotational broadening of TAP35 which both promotes blending and depresses the pseudo-continuum in regions of numerous weak lines. The final selection contains $25 \mathrm{Fe} I$ lines, ranging in excitation potential from 1.0 to $4.5 \mathrm{eV}$, and in landé value from 0.0 to 2.5 . We measured the equivalent width of each line in both TAP35 and $\tau$ Ceti by the following procedure. For TAP35, the continuum level was determined by eye on both sides of the profile, and a straight line was drawn between the two, under which the area of the line was computed by numerical integration. We preserved plots showing the regions used for all continuum placements so that identical placement could be employed for $\tau$ Ceti, thereby minimizing human bias in the measurements for the two stars. (We found that any "automatic" continuum determination occasionally erred owing to the large rotational broadening of TAP35.) Before carrying out the measurement of equivalent widths for $\tau$ Ceti, we convolved its spectrum with the rotational broadening function (Gray, 1974) with $v \sin i=17 \mathrm{~km} \mathrm{~s}^{-1}$ so that the continuum placement and weak blends affected the measurements in the same way as TAP35. Indeed, the resulting broadened spectrum of $\tau$ Ceti was remarkably similar to that of TAP35, though the Fe lines were clearly weaker in $\tau$ Ceti, an old disk star.

\section{Zeeman analysis}

\subsection{Basic approach}

The basic idea in this Zeeman analysis is to detect an enhancement of the equivalent widths of Zeeman-sensitive lines over those expected if no magnetic field existed. We model the line-transfer of all 25 lines self-consistently to predict their equivalent widths as a function of the surface magnetic field strength, $B$. The line-transfer technique has been described previously in detail (Basri and Marcy, 1988). Briefly, we calculate the LTE line transfer in all Stokes parameters, thus treating the Zeeman-induced polarization across each line profile. We construct model atmospheres for both TAP35 and $\tau$ Ceti by scaling $\mathrm{T}(\tau)$ of the solar atmosphere (Holweger and Müller, 1974) to the respective effective temperatures (see below) of the two stars and recomputing the densities and ionization equilibria to satisfy self-consistently hydrostatic equilibrium and the Saha equation (Gray, 1976).

\subsection{Oscillator strengths}

In principle, the proper procedure is to adopt laboratory $g f$ values (Fuhr et al., 1988; Blackwell et al., 1982) and to construct a model stellar atmosphere which minimizes the differences between observed and calculated equivalent widths. Instead, we construct a model atmosphere for $\tau$ Ceti, scaled from the HolwegerMüller solar model, and calculate empirical $g f$ values by forcing agreement between calculated and observed equivalent widths.

To do this, we adopt atmospheric parameters for $\tau$ Ceti from the fine analysis by Arribas and Crivellari (1989). However, we find that using their value of $\mathrm{Fe}$ abundance and lab $g f$ values yields calculated values of $W_{\text {eq }}$ that are systematically 
too low by $\sim 20 \%$. A similar effect in the same direction was found by Arribas and Crivellari when they used the Holweger-Müller solar model. So, we have derived a new Fe abundance for $\tau$ Ceti which, when using lab $g f$ values and scaled HolwegerMüller atmosphere, yields calculated equivalent widths that agree on average with those observed. The final $\mathrm{Fe}$ abundance for $\tau$ Ceti is: $\log \mathrm{Fe} / \mathrm{H}=-4.81$ (solar case, $\log \mathrm{Fe} / \mathrm{H}=-4.5$ ). We do not recompute the atmosphere itself for $\tau$ Ceti using this lower abundance since it makes little difference in predicted equivalent widths (Arribas and Crivellari, 1989). Final $g f$ values were then determined by trying a range of values for each line and determining the value that gave exact agreement between calculated and observed equivalent width for $\tau$ Ceti. We note that these empirical $g f$ values were found to be correlated with line strength, probably indicating an error in the Holweger-Müller atmosphere model. (Thanks are due to $J$. Valenti for pointing this out.)

\subsection{Zeeman enhancement in TAP35}

We computed the line transfer in TAP35 using an atmosphere and Fe abundance determined from the 10 lines having lowest sensitivity to the Zeeman effect. We computed the equivalent widths of these lines for a grid of cases spanning $T_{\text {eff }}$ and $\mathrm{Fe} / \mathrm{H}$, and determined (by interpolation) the values that yielded the best fit to the observed equivalent widths. The resulting values were: $T_{\text {eff }}=5250 \pm 100 \mathrm{~K}$ and $\log \mathrm{Fe} / \mathrm{H}=-4.21$. Thus, TAP35 is metal $\mathrm{r} \mathrm{ich},[\mathrm{Fe} / \mathrm{H}]=+0.29$, and has an effective spectral type of G8. We note that the determination of $T_{\text {eff }}$ was greatly aided by the following diagnostic. We demanded that the residuals between observed and calculated $W_{\text {eq }}$ be uncorrelated with excitation, $\chi_{\mathbf{e x}}$, since any such correlation implies a systematic error in photospheric temperature.

We now consider all 25 lines and compute the equivalent widths for TAP35 for a range of assumed magnetic field strengths. Surprisingly, the Zeeman enhancement of equivalent width does not monotonically increase with Landé factor, $g_{\mathrm{eff}}$, or with $\lambda^{2} g_{\text {eff }}$. The reason for this is that a complicated saturation effect takes place when multiple Zeeman components overlap, as is the case for $B=0 \mathrm{G}$. As one considers increasing field strengths, the Zeeman components begin to separate, diminishing the saturation. The rate of growth (with increasing $B$ ) of equivalent width is greatest when the magnetic field is strong enough for the components to become partially resolved. The critical issue is the separation of components having the same polarization. The result is that Zeeman $W_{\text {eq }}$-enhancement depends not only on Landé factor but also on the distribution of many $\pi$ and $\sigma$ Zeeman components over the profile thereby modulating the polarization saturation. Ironically, the simple Zeeman triplet, so useful for circular-polarization and Zeeman-broadening studies, experiences no further enhancement in $W_{\text {eq }}$ once the field strength is such that the components are fully separated.

Because of these effects, we develop a theoretical sensitivity index for Zeeman equivalent-width enhancement, defined as the ratio of the equivalent width for $B=1000 \mathrm{G}$ to that for $B=0 \mathrm{G}$. This index depends (weakly ) on the atmosphere under consideration, so we determine this index using the TAP35 atmosphere. Lines of highest sensitivity have equivalent-width ratios of 1.15-1.20 (i.e., $\lambda \lambda$ 6173, 
7491,8468 ), thus indicating that increases of $15 \%$ to $20 \%$ will occur as the field is increased from 0 to $1 \mathrm{kG}$. This demonstrates the ease with which strong, $\mathrm{kG}$ fields can be detected by this technique.

For TAP35, we plot in Fig. 1 the ratio of observed to theoretical $(B=0)$ equivalent widths versus the above Zeeman sensitivity index. The scatter in $W_{\text {eq }}(o b s) / W_{\text {eq }}(B=0)$ is about $10 \%$ (as seen in the lower left corner) and is due to three sources of error: measurement of equivalent widths, use of a scaled solar atmosphere for a star that undoubtedly has a perturbed photosphere, and our inadequate determination of $g f$ values. These sources of error are all unrelated to the Zeeman sensitivity of the individual lines. A weak trend is apparent in the plot such that the lines of highest Zeeman sensitivity indeed have observed $W_{\text {eq }}$ systematically larger than predicted for $B=0$. The correlation coefficient, 0.55 , implies a probability of less than $1 \%$ that the data are uncorrelated. We therefore conclude that TAP35 has a magnetic field exhibited by the enhancement in $W_{\text {eq }}$ of the most Zeeman-sensitive lines.

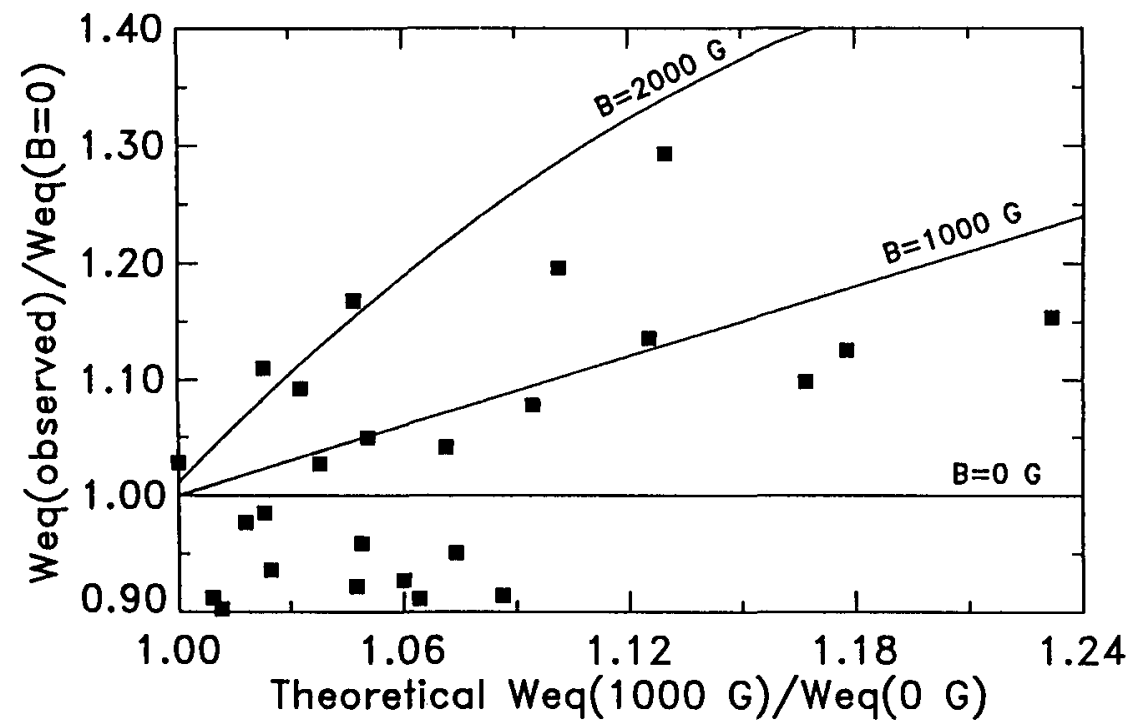

Fig. 1. Ratio of observed $W_{\text {eq }}$ to that predicted for $B=0$ vs. sensitivity to Zeemanenhancement, for 25 lines in the T Tauri Star, TAP35. Lines show expected (theoretical) locus of points for $B=0,1000$ and $2000 \mathrm{G}$.

We now calculate the locus of points expected on Fig. 1 for three different magnetic field strengths, 0,1000 , and $2000 \mathrm{G}$. This is done by synthesizing each line and assuming that the entire surface is covered by a specific field strength. The resulting theoretical loci are shown on Fig. 1. The seven most Zeeman-sensitive absorption lines (located in the right half of the figure) all lie above the locus line representing $B=0$, supporting the suggestion of magnetically-induced $W_{\text {eq }}$ enhancement. These seven points, however, all sit below the locus representing $B=2000 \mathrm{G}$, thus suggesting that the field is less than $2000 \mathrm{G}$, assuming a filling 
factor of $100 \%$. Preliminary tests show that the quantity best revealed by this technique of Zeeman-analysis is the product of strength and filling factor, $B f$. Thus, Fig. 1 suggests that $0.5 \mathrm{kG}<B f<2 \mathrm{kG}$.

\section{Conclusions}

The enhancement of equivalent widths of Zeeman-sensitive lines over insensitive lines serves as a diagnostic of magnetic fields, especially for stars having large rotation rates or uncertain atmospheres. Application to the weak T Tauri star, TAP35, reveals such Zeeman enhancement, and is consistent with an unsigned surface flux, $B f \approx 1 \mathrm{kG}$. Future refinements will include improved $g f$ values and semi-empirical model photospheres for $\mathrm{T}$ Tauri stars.

We would like to acknowledge J.A. Valenti for his assistance. We acknowledge partial support for this research from NSF grants AST-8919634 (to GM) and AST-8911596 (to GB).

\section{References}

Arribas, S., Crivellari, L.: 1989, Astron. Astrophys. 210, 211

Basri, G.: 1990, in High Resolution Spectroscopy in Astrophysics, a special issue of Memorie S. A. Ital., ed. R. Pallavicini

Basri, G., Marcy, G.W.: 1988, Astrophys. J. 330, 274

Blackwell, D.E., Petford, A.D., Simmons, G.J.: 1982, Mon. Not. R. Astron. Soc. 210, 595

Brown, D., Landstreet: 1981, Astrophys. J. 246, 899

Cram, L.: 1979, Astrophys. J. 234, 949

Calvet, N. et al: 1984, Astrophys. J. 277, 725

Cohen, M., Beiging, J.H.: 1986, Astron. J. 92, 1396

Finkenzeller, U., Basri, G.: 1987, Astrophys. J. 318, 823

Fuhr, J.R., Martin, G.A., Wiese, W.L.: 1988, J. Phys. and Chem. Ref. Data 17, No. 4

Gray, D.F.: 1976, in The Observation and Analysis of Stellar Photospheres, Wiley

Hartmann, L., Hewitt, R., Stahler, S., Mathieu, R.D.: 1986, Astrophys. J. 309, 275

Herbig, G.H.: 1970, Mem. Roy. Soc. Sci. Liege 19, 13

Holweger, H.: 1967, Z. Astrophys. 65, 365

Holweger, H., Müller, E.A.: 1974, Solar Phys 39, 19

Imhoff, C., Giampapa, M.: 1982, in Advances in Ultraviolet Astronomy: four Years of IUE Res., ed. Y. Kondo, J.M. Mead, R.D. Chapman (NASA CP 2338), p. 456

Johnstone, R.M., Penston, M.V.: 1986, Mon. Not. Roy. Ast. Soc. 218, 927

Johnstone, R.M., Penston, M.V.: 1987, Mon. Not. Roy. Ast. Soc. 227, 797

Kurucz, R.L., Furelid, I., Brault, J., Testerman, L.: 1984, Solar Flux Atlas from 269 to $1300 \mathrm{~nm}$, National Solar Observatory Atlas No. 1

Marcy, G.W., Basri, G.: 1989, Astrophys. J. 345, 480

Saar, S.: 1988, Astrophys. J. 324, 441

Shu, F.: 1990, private communication

Vrba, F.J., Rydgren, A.E., Chugainov, P.F., Shakhovskaya, N.I., Weaver, W.B.: 1989, Astron. J. 97, 483

Walter, F.M.: 1987, Pub. Ast. Soc. Pacific 09, 31

Walter, F.M.: 1988, Astron. J. 96, 297 